\title{
L-carnitine ameliorated fatty liver in high-calorie diet/STZ-induced type 2 diabetic mice by improving mitochondrial function
}

\author{
Yunqiu Xia ${ }^{1 \dagger}$, Qing $\mathrm{Li}^{2 \dagger}$, Weizhen Zhong ${ }^{1}$, Jing Dong ${ }^{3 *}$, Zhulin Wang ${ }^{2}$ and Chunbo Wang ${ }^{1}$
}

\begin{abstract}
Background: There are an increasing number of patients suffering from fatty liver caused by type 2 diabetes. We intended to study the preventive and therapeutic effect of $L$-carnitine $(L C)$ on nonalcoholic fatty liver disease (NAFLD) in streptozotocin (STZ)-induced type 2 diabetic mice and to explore its possible mechanism.

Methods: Thirty male Kungming mice were randomly divided into five groups: control group, diabetic group, pretreatment group (125 mg/kg BW), low-dose (125 mg/kg BW) therapeutic group and high-dose (250 mg/kg BW) therapeutic group. The morphology of hepatocytes was observed by light and electron microscopy. LC and ALC (acetyl L-carnitine) concentrations in the liver were determined by high-performance liquid chromatography (HPLC). Moreover, liver weight, insulin levels and free fatty acid (FFA) and triglyceride (TG) levels in the liver and plasma were measured.

Results: Average liver LC and ALC levels were $33.7 \%$ and 20\% lower, respectively, in diabetic mice compared to control mice $(P<0.05)$. After preventive and therapeutic treatment with $L C$, less hepatocyte steatosis, clearer crista and fewer glycogen granules in the mitochondria were observed. Decreased liver weight, TG levels, and FFA concentrations $(P<0.05)$ in the liver were also observed after treatment with LC in diabetic mice. Moreover, liver $L C$ and $A L C$ levels increased upon treatment with $L C$, whereas the ratio of $L C$ and ALC decreased significantly $(P<$ 0.01).
\end{abstract}

Conclusion: LC supplements ameliorated fatty liver in type 2 diabetic mice by increasing fatty acid oxidation and decreasing the LC/ALC ratio in the liver. Therefore, oral administration of LC protected mitochondrial function in liver.

Keywords: Type 2 Diabetes Mellitus, Carnitine, Nonalcoholic Fatty Liver Disease (NAFLD), Mitochondria

\section{Introduction}

Nonalcoholic fatty liver disease (NAFLD), one of the most common complications of type 2 diabetes, is characterized by an increase in fatty acids, triglycerides, and cholesterol levels [1] and fat accumulation in the liver. Among type 2 diabetes patients, $50 \%-70 \%$ of individuals were diagnosed with NAFLD; in obese patients, that value increases to $95 \%$ [2]. Recent studies have shown that NAFLD and insulin resistance [3] were involved in

\footnotetext{
* Correspondence: jingdong8@yahoo.com.cn

† Contributed equally

${ }^{3}$ Physiology Department of the Medical College, Qingdao University, Ningxia Road, Qingdao, China

Full list of author information is available at the end of the article
}

metabolic syndrome (MS), especially in fatty acid metabolic disorder, which primarily occurred in obese and type 2 diabetes patients [4]. Prolonged exposure to free fatty acids damages pancreatic $\beta$-cells and hepatocytes [5]. Furthermore, excessive fat accumulation in the liver damages mitochondria, which are the primary cellular sites for fatty acid utilization [6,7]. Current general therapies to treat the early stages of fatty liver disease include lifestyle modification approaches such as exercise and weight loss with diet. The goals of these strategies are to normalize aminotransferase levels and to reduce liver fat levels and inflammation. However, none of these strategies specifically improve mitochondrial function in type 2 diabetes patients. The aim of the

\section{() Biomed Central}


current study was to find treatments that would improve mitochondrial function in fatty liver disease in type 2 diabetes patients.

L-Carnitine (L- $\beta$-hydroxy- $\gamma$ - $\mathrm{N}$-trimethylaminobutyric acid $[8,9])(\mathrm{LC})$ is present in the free or acyl-carnitine form in the plasma [10]. LC plays an important role in lipid metabolism; it acts an essential cofactor for the $\beta$ oxidation of fatty acids by facilitating the transport of long-chain fatty acids across the mitochondrial membrane as acyl-carnitine esters. It can activate carnitine palmityl transferase-1 (CPT-1), the key enzyme in fatty acid oxidation [11]. Furthermore, because LC shuttles acetyl groups from inside to outside the mitochondrial membrane, it increases the $\mathrm{CoA}-\mathrm{SH}$ /acetyl-CoA ratio in the mitochondrion by forming Acetyl L-carnitine (ALC) with the help of carnitine acetyltransferase (CAT) $[12,13]$, whose activity relieves inhibition of the $\mathrm{PDH}$ complex and increases glycometabolism [14-16]. According to a previous study, significantly reduced lactate plasma levels suggested that LC can also stimulate the activity of pyruvate dehydrogenase (PDH) [17], whose activity is deficient in type 2 diabetic patients [18]. These results demonstrated that LC can act as a carrier of acetyl groups from the mitochondria to the cytosol $[15,19]$. In addition, LC was defined as a biomarker to assess the function of the mitochondria [20]. Evidence showed that a combination of nutrients, including LC, can improve mitochondrial dysfunction in the liver of type 2 diabetic Goto-Kakizaki rats [21]. Recently, Karanth et al. proposed that LC supplements enhanced the activity of mitochondrial enzymes such as CPT-1 and the respiratory chain enzymes [22]. Based on the evidence described above, we explored the effects of LC on NAFLD caused by type 2 diabetes.

The earliest study of the effect of LC on steatohepatitis was performed by Bowyer et al. in 1988 [23]. They claimed that LC deficiency was not the major cause of liver steatosis. However, a recent study showed that LC played a role in reducing steatosis in patients with hepatitis $C$ treated with IFN alpha and ribavirin [24]. LC supplements in patients with NASH (non-alcoholic steatohepatitis) greatly improved glucose plasma levels, lipid profiles and histological manifestations [25]. However, none of these studies focused on the effect of LC on NAFLD caused by type 2 diabetes, especially in diabetic mice.

The aim of the present study was to explore the preventive and therapeutic effect of LC on NAFLD in streptozotocin (STZ)- and diet-induced type 2 diabetic mice with regard to morphological and biochemical aspects. We also intended to determine whether its effect was due to an improvement in the mitochondrial function of hepatocytes to provide theoretical evidence for LC as a clinical therapy for NAFLD.

\section{Materials and methods \\ Animals}

Male Kunming SPF 3-week-old mice (Institute for Drug Control of Qingdao, China), weighing between $15 \mathrm{~g}$ and $17 \mathrm{~g}$ were used. They were fed a diet containing 59\% basic mice feed, $20 \%$ sugar, $18 \%$ lard, and 3\% egg yolk. They had free access to food and water in an animal room maintained at $22 \pm 3^{\circ} \mathrm{C}$. The protocols used for handling the mice were approved by the Qingdao University Center for human functional experiment of the medical college Animal Care Committee and were in accordance with the guidelines set by the National Institutes of Health Guide for Care and Use of Laboratory Animals.

The animals were randomly divided into five groups: a control group (only high-calorie diet), a diabetic group, a preventive treatment group $(125 \mathrm{mg} / \mathrm{kg}$, intragastric, i. g.) and two therapeutic groups $(250 \mathrm{mg} / \mathrm{kg}, 125 \mathrm{mg} / \mathrm{kg}$, i.g.). At the age of 6 and 9 weeks, two low doses of STZ (100 mg/kg, i.p., provided by Sigma) prepared in $0.1 \mathrm{~N}$ citrate buffer at $\mathrm{pH} 4.5$ were given to mice in the diabetic, preventive and therapeutic groups. Age-matched control group mice were injected with NS (normal saline) alone. Mice with fasting blood glucose levels above $12 \mathrm{mmol} / \mathrm{L}$ and insulin levels within the average range of the control group were considered type 2 diabetic mice.

\section{Drugs}

LC (provided by Northeast Pharmaceutical Factory, China) was dissolved in double distilled water. In the study of the preventive effect of LC on hepatopathy in type 2 diabetic mice, oral administration of LC (lowdose, $125 \mathrm{mg} / \mathrm{kg}$, i.g.) began on the day the mice were provided the high-calorie diet (3 weeks of age). LC was administrated to the therapeutic groups after they had developed diabetes (9 weeks of age). The two therapeutic groups of diabetic mice were given different oral doses of LC, a high-dose (250 mg/kg, i.g.) and a lowdose (125 mg/kg, i.g.). All the administrations were given once a day until the mice were 12 weeks of age. In addition, $\mathrm{LC}$ was administrated to mice in the preventive and therapeutic groups $30 \mathrm{~min}$ before samples were collected. Mice in the control group were given the same volume of saline. (Table 1: experimental design)

\section{Preparation of plasma and liver homogenates}

All mice were sacrificed at 12 weeks of age. Blood samples were collected via retro-orbital bleeding. Fresh livers were observed and weighed. Livers were homogenized, and $1 \mathrm{ml}$ of supernatant was collected and stored at $-20^{\circ} \mathrm{C}$ until analyzed. 
Table 1 Experimental design

\begin{tabular}{|c|c|c|c|}
\hline Group & 3 weeks old & 6 weeks old & 9 weeks old \\
\hline Control & $\mathrm{HFD+NS}$ & HFD+NS (CB) & HFD+NS (CB) \\
\hline DM+NS & $\mathrm{HFD+NS}$ & HFD+NS (STZ) & $\mathrm{HFD+NS}(\mathrm{STZ})$ \\
\hline Pre-treat & $\mathrm{HFD}+\mathrm{LC}$ & HFD+LC (STZ) & HFD+LC (STZ) \\
\hline $\mathrm{DM}+\mathrm{LC}(\mathrm{L})$ & HFD+NS & HFD+NS (STZ) & HFD+LC (STZ) \\
\hline $\mathrm{DM}+\mathrm{LC}(\mathrm{H})$ & HFD+NS & HFD+NS (STZ) & $\mathrm{HFD}+\mathrm{LC}(\mathrm{STZ})$ \\
\hline
\end{tabular}

Control: control group $(n=6), D M+N S$ : diabetic group $(n=6)$, pre-treatment: preventive group $(\mathrm{n}=6), \mathrm{DM}+\mathrm{LC}(\mathrm{L})$ : low-dose therapeutic group $(125 \mathrm{mg} / \mathrm{kg}$ i.g.) $(n=6), D M+L C(H)$ : high-dose therapeutic group $(250 \mathrm{mg} / \mathrm{kg}$, i.g. $)(n=6)$. All administrations of LC were given orally. HFD: high-fat diet; STZ:

streptozotocin; LC: L-carnitine; CB: citrate buffer. At the age of 6 and 9 weeks, mice were injected twice with $\mathrm{STZ}$ or $\mathrm{CB}$, respectively.

\section{Liver and body weights}

Body weights were measured once a week with an electric balance (Mettler Toledo, PL1501-S, Shanghai, China). Whole explanted livers were weighed on an electric analytical balance (Mettler Toledo, AL104, Shanghai, China). Relative liver weights (per body weight) were calculated (relative liver weight: liver weight/body weight $\times 100 \%$ ).

\section{Liver morphology}

Samples were washed with phosphate-buffered saline and fixed with $4 \%$ paraformaldehyde. Longitudinal sections $(10 \mu \mathrm{m})$ were cut in a microtome (model CM1900; Leica, Germany), placed on microscope slides and stained with oil red O. Image Pro Plus 6.0 was used to quantify the size of the lipid droplets. For electron microscopy analysis, liver apexes were processed according to routine procedures [26]. Ultrastructural changes in the liver were observed by transmission electron microscopy (TEM) (provided by JEOL, 1200EX).

\section{Measurement of triglyceride and FFA concentrations in the plasma and liver}

Liver free fatty acid levels were estimated by a $\mathrm{Cu}$-colorimetric method using a non-esterified fatty acid assay kit (provided by Applygen Technologies Inc. Beijing, China). The results are expressed in absorbance values. TG levels in the plasma and liver were determined by photoelectric colorimetry using an automatic biochemical analyzer (Saturno-300, provided by CRNOY S.R.I Company, Italy).

\section{LC and ALC concentrations in the liver}

The concentrations of LC and ALC in the liver were analyzed by high-performance liquid chromatography (HPLC). This method was first established in our lab [27]. A Waters2695 High Performance Liquid Chromatography system (Waters, America) equipped with a Hypersil C18 column $(5 \mu \mathrm{m}$ particle size, $200 \mathrm{~mm}$ length, $4.6 \mathrm{~mm}$ internal diameter) and a Waters 474 fluorescence detector (Waters, America) was used.

\section{Statistical analysis}

The data are expressed as the mean \pm SD. Statistically significant differences between groups were assessed by analysis of variance (one-way ANOVA), repeated measures and multivariate tests followed by LSD. A P value of less than 0.05 was considered statistically significant.

\section{Results}

\section{Changes in blood glucose and plasma insulin levels}

Blood glucose levels were 197\% higher in the diabetic group than in the control group (control+NS $6.35 \pm$ $0.63 \mathrm{mmol} / \mathrm{L}$ vs. $\mathrm{DM}+\mathrm{NS} 18.9 \pm 6.04 \mathrm{mmol} / \mathrm{L}, \mathrm{P}=$ $0.001)$. Plasma insulin levels in diabetic mice were normal (control+NS $12.94 \pm 0.91 \mathrm{mIU} / \mathrm{L}$ vs. DM+NS 11.56 $\pm 1.09 \mathrm{mIU} / \mathrm{L}, \mathrm{P}=0.503)$.

\section{Effects of LC on changes in body weight in STZ-treated mice}

At the 8th week, the average body weight of the preventive group was $14.6 \%$ lower than in the control group (control group $40.617 \pm 2.919$ g vs. preventive group $34.7 \pm 2.571 \mathrm{~g}, \mathrm{P}=0.016$ ). From the 10 th week on, significant differences in body weight between the preventive and diabetic groups were apparent $(P=0.012)$. At the end of the 12th week, mice in the two therapeutic groups were heavier than those in the diabetic group (Figure 1).

\section{Lipid metabolism}

All diabetic mice had higher liver FFA $(+22.6 \%)$ and TG $(+402.6 \%)$ levels, plasma TG levels $(+225 \%)$, liver weight $(+84.6 \%)$ and relative liver weight $(+50 \%)$.

As shown in table 2, after 3 and 6 weeks of treatment with oral LC, liver FFA levels decreased in the pre-treatment, high-dose and low-dose therapeutic groups. There was no significant difference in FFA levels between these mice and mice in the control group $(P>0.175)$. LC therapy also remarkably decreased liver TG levels (DM + NS vs. pre-treatment $\mathrm{P}<0.01, \mathrm{DM}+\mathrm{NS}$ vs. high-dose $\mathrm{P}<0.01, \mathrm{DM}+\mathrm{NS}$ vs. low-dose $\mathrm{P}<0.05)$. Moreover, the mean plasma TG levels were $1.2 \pm 0.1 \mathrm{mmol} / \mathrm{L}$ in the control group and $3.9 \pm 2.3 \mathrm{mmol} / \mathrm{L}$ in the diabetic group. LC caused a decreased in plasma TG levels, especially in the high-dose and preventive treatment groups (high-dose therapeutic group $1.625 \pm 0.399 \mathrm{mmol} / \mathrm{L}$, preventive group $1.713 \pm 0.636 \mathrm{mmol} / \mathrm{L}$ ).

The average liver weight in the diabetic group was $44.6 \%$ higher than in the control group (table 2) (control $0.0439 \pm 0.0050$ vs. DM+NS $0.0635 \pm 0.0103, \mathrm{P}<0.01)$. After administrating LC for prevention and treatment purposes, the liver weight significantly decreased. After treatment with $\mathrm{LC}$, the relative liver weight also decreased, both in the high-dose therapeutic group $(0.0489 \pm 0.0020, \mathrm{P}<0.01$ vs. diabetic group $)$ and in the 


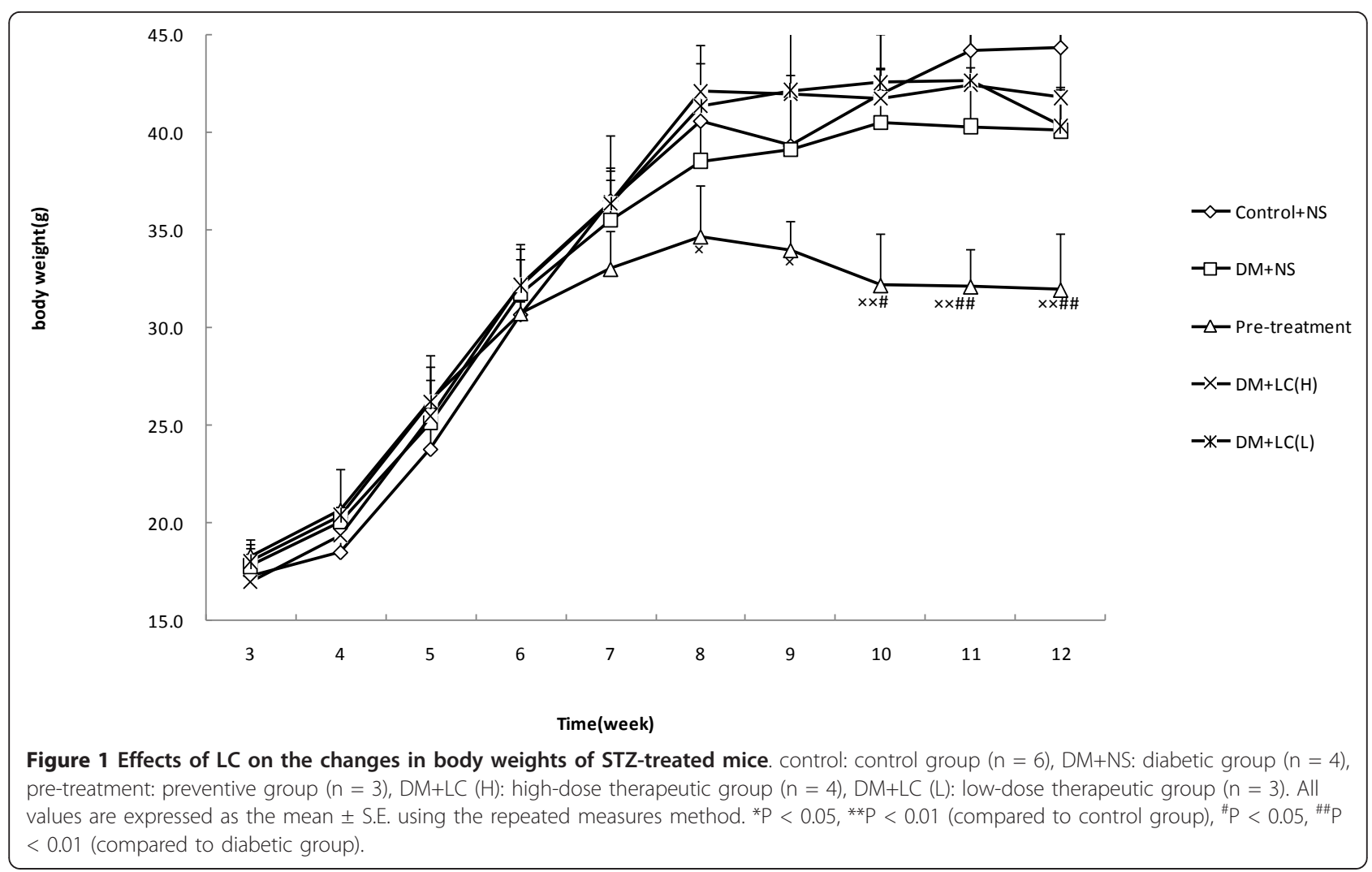

low-dose therapeutic group $(0.0544 \pm 0.0031, \mathrm{P}<0.05$ vs. diabetic group). Furthermore, the effect of LC on liver weight was dose dependent.

\section{LC and ALC system}

Mean LC and ALC levels were $33.7 \%$ and 20\% lower in the diabetic group than in the control group, respectively (LC P $=0.033$ ) (table 2 ). Mice in the preventive and therapeutic groups expressed higher levels of LC than those in the diabetic group, which were not significantly different than those of the control group. In total, the exogenous administration of LC could compensate for the deficiency of both LC and ALC in type 2 diabetic mice. Interestingly, average LC concentrations in the high-dose therapeutic group were lower than in the low-dose therapeutic group, whereas the high-dose therapeutic group expressed the highest ALC levels among the five groups (table 2). There were no significant differences in the LC/ALC ratio between the control and diabetic groups (Control+NS $16.9455 \pm 4.7270 \mu \mathrm{mol} / \mathrm{L}$, $\mathrm{DM}+\mathrm{NS} 16.5659 \pm 5.9218 \mu \mathrm{mol} / \mathrm{L}$ ), whereas the ratio decreased after LC treatment. Among all the therapeutic groups, the ratio in the high-dose group was the lowest. The differences in the LC/ALC ratio between high-dose

Table 2 Metabolic parameters

\begin{tabular}{|c|c|c|c|c|c|}
\hline Variable & Control & $\mathrm{DM}+\mathrm{NS}$ & Pre-treatment & $\mathrm{DM}+\mathrm{LC}(\mathrm{L})$ & $\mathrm{DM}+\mathrm{LC}(\mathrm{H})$ \\
\hline Body Weight (g) & $44.4 \pm 3.4$ & $40.1 \pm 2.1$ & $32.0 \pm 2.8^{\text {x॥\#\# }}$ & $40.3 \pm 2.0$ & $41.8 \pm 5.6$ \\
\hline Liver weight (g) & $1.3 \pm 0.3$ & $2.4 \pm 0.5^{* *}$ & $2.0 \pm 0.4^{*}$ & $1.9 \pm 0.3^{* \#}$ & $1.8 \pm 0.2^{\#}$ \\
\hline Relative liver weight & $0.04 \pm 0.01$ & $0.06 \pm 0.01^{* *}$ & $0.06 \pm 0.01$ & $0.05 \pm 0.00^{\#}$ & $0.05 \pm 0.00^{\# \#}$ \\
\hline FFA in liver $(L / g \cdot c m)$ & $1.7 \pm 0.1$ & $2.0 \pm 0.3^{*}$ & $1.8 \pm 0.2$ & $1.9 \pm 0.3$ & $1.8 \pm 0.1$ \\
\hline TG in liver (mmol/L) & $0.23 \pm 0.04$ & $1.16 \pm 0.6^{* *}$ & $0.34 \pm 0.2^{\# \#}$ & $0.55 \pm 0.09^{\#}$ & $0.48 \pm 0.1^{\# \#}$ \\
\hline TG in plasma (mmol/L) & $1.2 \pm 0.1$ & $3.9 \pm 2.3^{* *}$ & $1.7 \pm 0.6$ & $2.2 \pm 1.0$ & $1.6 \pm 0.4$ \\
\hline LC in liver $(\mu \mathrm{mol} / \mathrm{L})$ & $16.9 \pm 3.4$ & $11.2 \pm 2.6^{*}$ & $12.1 \pm 3.1$ & $14.9 \pm 1.9$ & $12.6 \pm 4.1$ \\
\hline ALC in liver $(\mu \mathrm{mol} / \mathrm{L})$ & $1.0 \pm 0.2$ & $0.8 \pm 0.3$ & $1.0 \pm 0.7$ & $1.4 \pm 0.2$ & $2.3 \pm 0.9^{* * \# \#}$ \\
\hline LC/ALC & $16.9 \pm 4.7$ & $16.6 \pm 5.9$ & $15.4 \pm 6.6$ & $11.3 \pm 2.8$ & $5.6 \pm 1.5^{* * \# \#}$ \\
\hline
\end{tabular}

Control: control group, DM+NS: diabetic group, pre-treatment: preventive group, DM+LC (L): low-dose therapeutic group, DM+LC (H): high-dose therapeutic group. All values are expressed as the mean \pm S.E. using the repeated measures method. ${ }^{*} P<0.05,{ }^{* * P}<0.01$ (compared to control group), $P$ P $<0.05$, ${ }^{\# \# ~} P<0.01$ (compared to diabetic group). 
group and control and diabetic groups were both significant $(\mathrm{P}<0.01)$.

\section{Morphological changes in the liver}

The size of the livers in the diabetic mice was generally larger than in control mice. In general, the livers of diabetic mice were yellow in color with numerous particles on the surface and were much harder than normal livers. After treatment with $\mathrm{LC}$, the size of the livers decreased, and the surfaces became smoother.

To analyze fat deposits in liver cells, liver sections were stained with oil red $\mathrm{O}$. Obvious liver fatty degeneration and piecemeal necrosis of hepatocytes were observed in the diabetic group by LM (Figure 2-a). There were many red lipid deposits (stained by red oil O) in the hepatocytes of the diabetic group. As shown in Figure 2-I, the number of lipid deposits decreased with increasing doses of LC, especially in the preventive and high-dose therapeutic groups. Images from these two groups are very similar to those of the control group. As shown in Figure 2-II, the percentage of the lipid profile area to total area in diabetic mice was $5.44 \%$, which is consistent with a clinical diagnosis of NAFLD [28]. Although the lipid area in the low-dose therapeutic mice was still fairly high $(2.0 \pm 0.52 \%)$, it was significantly lower than in the diabetic mice $(\mathrm{P}<$ 0.01 ). With the increasing dose of LC or the extension of treatment time, the lipid area percentage (Figure 2-b) decreased by $95.8 \%$ in the high-dose therapeutic group and $92.6 \%$ in pre-treatment group. There was no significant difference in lipid area percentage between mice in the LC treatment groups and the control group.

Obvious mitochondrial swelling, crista disorder and an increase in electron density were observed by TEM in the diabetic group (Figure 3B) compared to the control group (Figure $3 \mathrm{~A}$ ). In addition, there were more glycogen granules in the cytoplasm in the diabetic group, which is a characteristic of type 2 diabetes. Morphological damage of the mitochondria recovered after administration of LC. The LC groups possessed clearer crista (Figure 3C-E) and rough endoplasmic reticulum (Figure $3 \mathrm{C})$ with dense granules.

\section{Discussion \\ Model}

Results from another study in our lab clearly indicated that we successfully created type 2 diabetic mice through a combination of a high-calorie diet and two low-dose STZ injections. Although the insulin levels of the diabetic mice were normal, the blood glucose levels were high. These results were consistent with characteristics of type 2 diabetes [29]. To verify the diagnosis of NAFLD, a liver biopsy, which is the gold standard [28], was performed. The biopsy revealed that the lipid area was above 5\% (Figure 2-b). Moreover, liver TG and FFA levels of diabetic mice were increased. All of these results are consistent with characteristics of type 2 diabetes: high glucose, insulin resistance, hyperlipidemia and obesity. Therefore, type 2 diabetes can be induced in mice by a combination of a high-calorie diet and two low-dose STZ injections to study the pathophysiological changes caused by diabetes or to evaluate the therapeutic effects of LC on fatty liver in type 2 diabetes.

\section{Morphological changes}

The prevalence of NAFLD is high in obesity, diabetes, high-calorie diet and hyperlipidemia [30]. Our research confirmed the characteristics of NAFLD in diabetic mice. The gross appearance of the liver improved upon treatment with LC; the liver decreased in size and appeared brighter and less greasy. These changes were partly observed by quantifying the lipid droplets by LM (Figure 2-b). Therefore, it could be concluded that LC improved steatosis of the liver in type 2 diabetic mice. There was no obvious fibrosis in these images, suggesting that the diabetic mice in our study only suffered from fatty liver and had not yet developed cirrhosis. Therefore, this process was reversible. In addition, there were large areas of glycogen granules in the cytoplasm of diabetic mice, which is the electron microscopy feature of type 2 diabetes. Large numbers of granules attached to the rough endoplasmic reticulum in the preventive group, also suggested that cell function significantly improved upon administration of LC. To ensure the therapeutic effect of LC from a general perspective, body weight and relative liver weight values were evaluated. These results suggest that LC stimulated fat metabolism and improved mitochondrial function in the liver.

\section{Mechanisms of liver dysfunction caused by type 2 diabetes mellitus}

It was hypothesized that in type 2 diabetes mellitus, there was a shift in substrate utilization from carbohydrates to lipids [31]. From what has been discussed above, we knew that type 2 diabetic mice had disorders in both glucose and lipid metabolism. Glucose metabolism in diabetic mice was insufficient, which could relieve the suppression of lipolysis. The lack of suppression of lipolysis increased the influx of fatty acids to the liver, leading to increased free fatty acid and triglyceride levels in the liver (table 2). The peripheral fat storage was then consumed, and the liver weight and relative liver weight increased. After large amounts of FFAs enter the liver, $\beta$-oxidation of fatty acids was stimulated, further inhibiting glycolysis [32]. Moreover, evidence showed that high levels of FFA could stimulate gluconeogenesis. At the same time, increased FFA and TG 


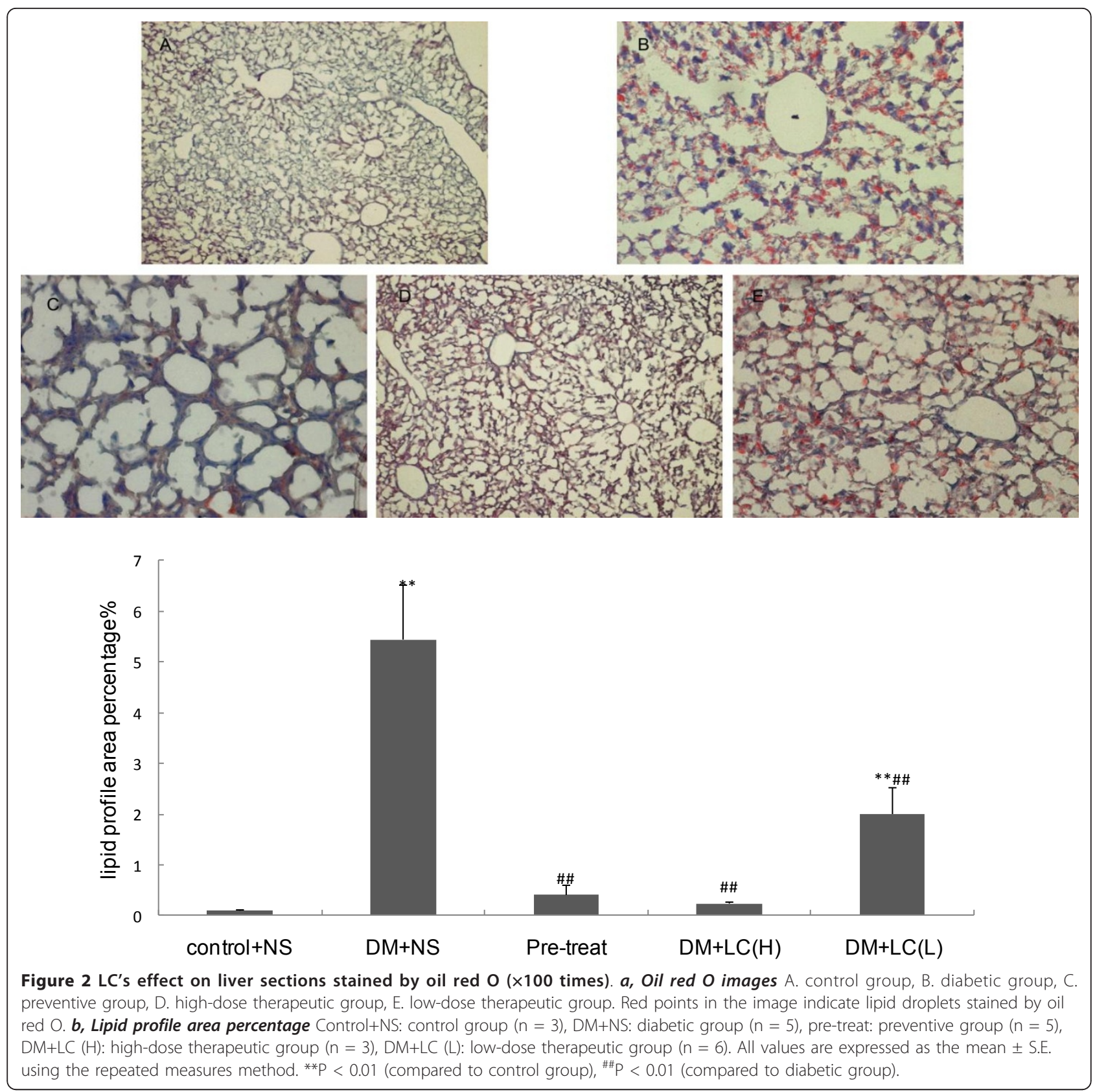

levels could increase the burden of the liver [33]. Although the activity of CPT-1, which is the rate-limiting enzyme in fatty acid oxidation, was elevated when large amounts of FFA enter the liver [11], the deficiency of LC in type 2 diabetes limits its activity. Therefore, the activity of CPT-1 is not sufficient to handle the elevated transport of fatty acids into the liver. All of these factors lead to excess accumulation of hepatic fat, including FFAs and TGs [34]. It was reported that TG stored in non-adipocyte cells led to cellular damage as a result of their lipotoxicity [35]. This process is shown in Figure 4.

\section{The effects of LC}

LC plays a critical role in fatty acid oxidation of energy regulation [36,37]. It serves as a carrier to facilitate the transport of long-chain fatty acids through the mitochondrial membrane and to undertake free fatty acid $\beta$-oxidation. Experimental evidence suggested that this step is a rate-limiting step in fatty acid oxidation. Diabetic mice had decreased liver LC levels (table 2), which is consistent with a previous study in which diabetic rats had significantly lower serum and liver LC levels than normal rats [38]. Administrating LC through 


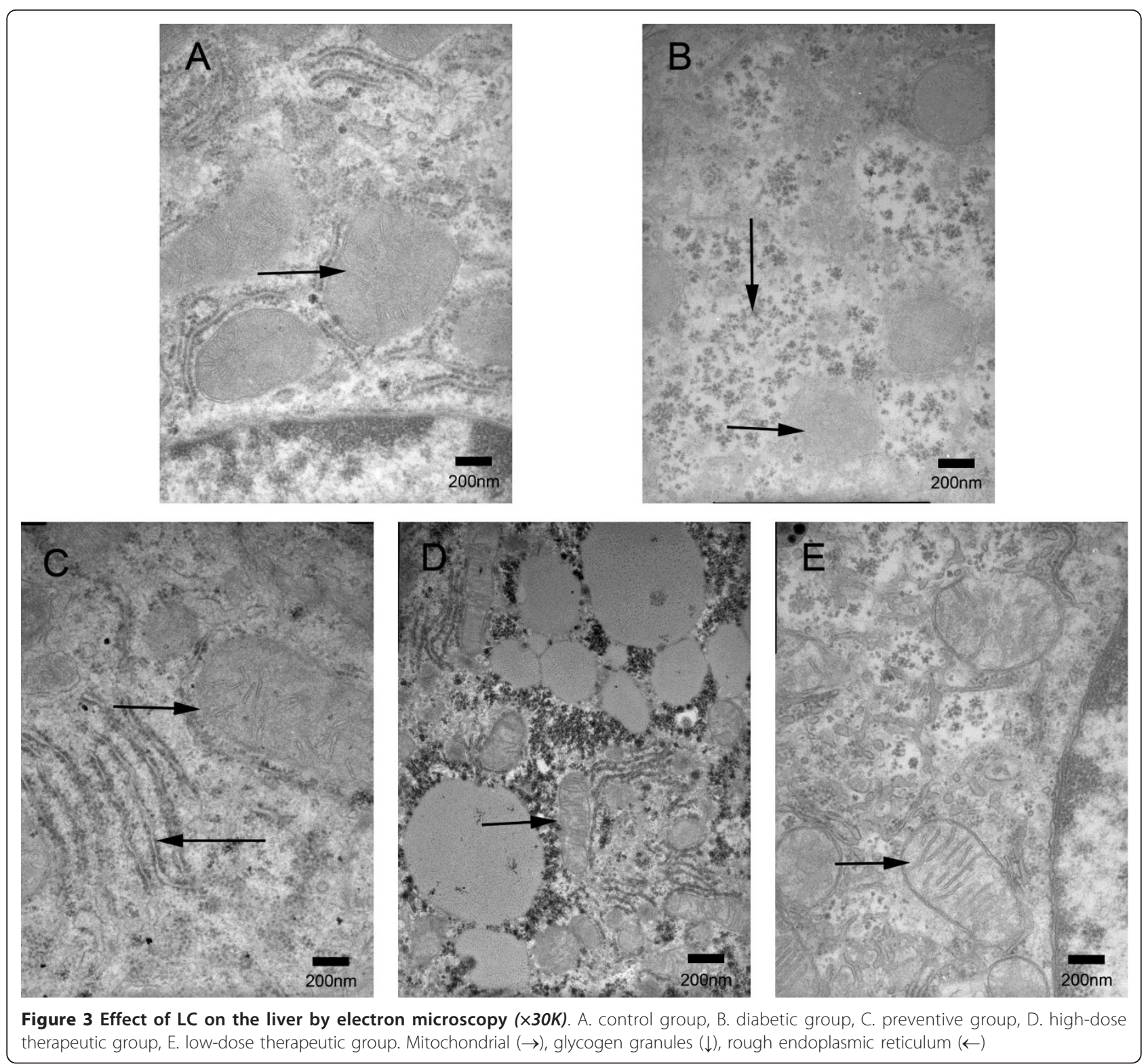

i.g. compensated for the deficiency of LC and ALC in the liver. As a result, fatty acid $\beta$-oxidation increased [39], and lipid accumulation in the liver decreased. Elevation of fatty acid $\beta$-oxidation decreased fat synthesis. Therefore, the amount of TG transported from the liver into the plasma decreased. Plasma insulin concentrations were significantly elevated after different doses of LC, which is consistent with other studies in our lab. A previous study showed that insulin inhibited the activity of HSL (hormone-sensitive triglyceride lipase), the key enzyme in lipolysis (Figure 4). Therefore, the amount of FFAs transported from the peripheral fat to the liver decreased. In this way, the decrease in body weight in diabetic mice could be prevented. It is interesting to note that if LC was administrated before the mice developed diabetes, it prevented weight gain in diabetic mice from an early stage. This indicates that the obesity burden could be prevented. Therefore, preventive administration of LC to high-calorie diet-treated mice maintained the energy balance and resisted the effect of STZ. Overall, LC helped lighten the burden of the liver and improve the symptoms of NAFLD caused by type 2 diabetes. LC could also relieve the harm of high TG levels to the cardiovascular system and could therefore be beneficial to diabetic patients. Moreover, when body weight and liver lipid concentrations were controlled at relatively low levels, blood glucose levels were low in mice in the pre-treatment group $(\leq 12 \mathrm{mmol} / \mathrm{L})$. This helped mice fed a high- 


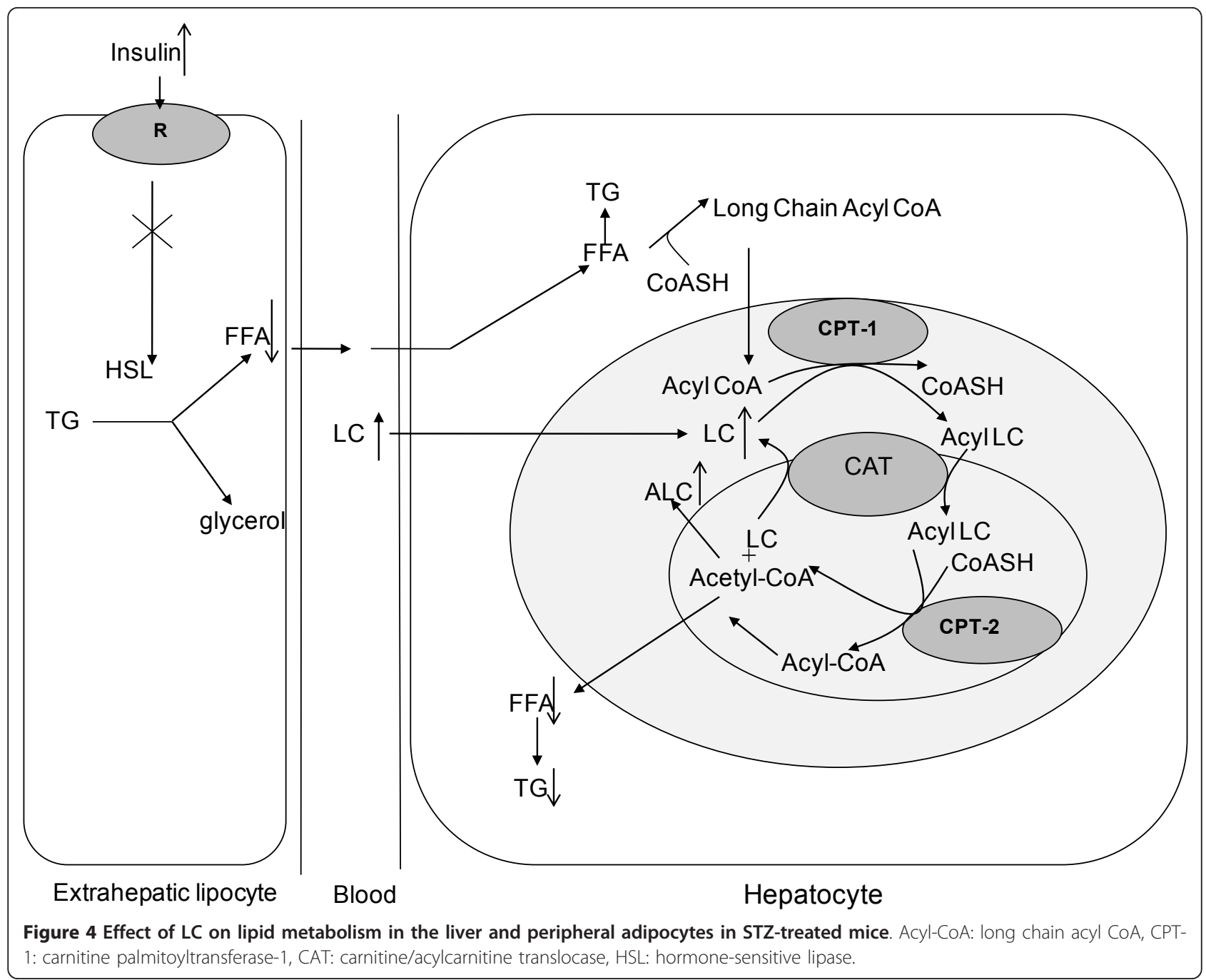

calorie diet resist the effects of STZ. Therefore, LC should be administrated in the early stages of diabetes.

Our study revealed that the ratio of LC to ALC decreased in the liver after $\mathrm{LC}$ administration even if both LC and ALC levels were markedly increased, which has not been observed before. According to a previous study, LC increases the CoA/acetyl-CoA ratio in the mitochondria by converting to ALC through the activity of CAT $[12,13]$. This process demonstrated that LC acted as a carrier of acetyl groups from the mitochondria to the cytosol $[15,19]$. In our study, ALC levels increased more in the high-dose treatment group, but LC concentrations in the low-dose therapeutic group were much higher than in the high-dose group. This result confirmed our hypothesis that exogenous LC supplements could shift the balance of LC + acetyl-CoA $\rightleftharpoons \mathrm{ALC}+\mathrm{CoA}$ to the right. Therefore, the concentrations of ALC and CoA were elevated. Previous data have shown that CoA was required for $\beta$ - oxidation and other energy metabolism pathways [16]. Therefore, LC helped to decrease the accumulation of acetyl-CoA in the mitochondrial matrix. For this reason, both glucose and lipid metabolism could be stimulated. In another study, excessive acetyl-CoA was the substrate of lipogenesis [40]. Therefore, LC could also inhibit TG produce in liver by decreasing acetylCoA levels. Furthermore, plasma TG transported from liver was decreased.

\section{Liver function improvement}

Disorders such as liver disease, defects in fatty acid metabolism, and the administration of pharmacological agents (e.g., pivampicillin or valproic acid) can cause secondary LC deficiency $[41,42]$. In our study, elevated FFA and TG accumulation causes lipotoxicity in hepatocytes, which could disrupt liver function. Mitochondrial dysfunction due to oxidative damage was previously detected in type 2 diabetes [21]. This dysfunction 
referred to a loss of capacity to synthesize LC and other proteins such as apolipoprotein and other useful factors. This aggravated damage to the liver and other organs. Exogenous administration of LC could compensate for this deficiency, which led to a beneficial cycle. Recently, DA Rossignol and colleagues used LC as a mitochondrial biomarker [20]. LC was indicated to be an essential factor in maintaining mitochondrial function. LC helped restore protein synthesis in mitochondrial membranes and showed an anti-oxidative effect $[21,43]$. The protective effect of LC on mitochondria was confirmed morphologically by EM (Figure 3). In conclusion, LC had an important mitochondrial detoxification effect on hepatocytes and other cells [41]. It improved symptoms of NAFLD and might improve metabolic conditions in type 2 diabetes mellitus.

\section{Conclusions}

Type 2 diabetes induced by a high-calorie diet and two low-dose STZ injections in mice resulted in decreased liver LC contents. LC supplements improved mitochondrial function in the liver by accelerating the transport of free fatty acids into the mitochondria and regulating the matrix ratio of LC to ALC. LC might be an effective liver protective medicine for delaying the progression of type 2 diabetes mellitus complications.

\section{List of abbreviations}

NAFLD: nonalcoholic fatty liver disease; LC: L-carnitine; ALC: acetyl Lcarnitine; STZ: streptozotocin; NASH: non-alcoholic steatohepatitis; LM: light microscopy; EM: electron microscopy; FFA: free fatty acid; TG: triglyceride; HPLC: high-performance liquid chromatography; CPT-1: carnitine palmityl transferase-1; CAT: carnitine acetyltransferase; PDH: pyruvate dehydrogenase; DM: diabetes mellitus; NS: normal saline.

\section{Acknowledgements}

This work was supported by the Qingdao Municipal of Science and Technology Commission Foundation (NO. 09-1-3-68-jch), Shandong, China; Shandong Province Natural Science Foundation (No. Y2008C60) and was partly supported by the National Natural Science Foundation of China, Grant No. 31071013

\section{Author details \\ ${ }^{1}$ Center for Human Functional Experiment, Medical College, Qingdao University, Ningxia Road, Qingdao, China. ${ }^{2}$ Class 5, Grade 2007, Medical College, Qingdao University, Ningxia Road, Qingdao, China. ${ }^{3}$ Physiology Department of the Medical College, Qingdao University, Ningxia Road, Qingdao, China.}

\section{Authors' contributions}

YQX performed the high-performance liquid chromatography experiments and helped draft the manuscript. QL participated in the design of the experiments, carried out the study and drafted the manuscript. JD participated in the design of the experiments, revised the manuscript critically and gave final approval of the version to be published. ZLW participated in the design of the experiments and analyzed and interpreted the data. WZZ participated in the acquisition and analysis of data. CBW participated in the design and coordination of experiments. All authors read and approved the final manuscript.

\section{Competing interests}

The authors declare that they have no competing interests.
Received: 22 September 2011 Accepted: 15 November 2011

Published: 15 November 2011

\section{References}

1. Marchesini G, Brizi M, Bianchi G, Tomassetti S, Bugianesi E, Lenzi M, McCullough AJ, Natale S, Forlani G, Melchionda N: Nonalcoholic fatty liver disease: a feature of the metabolic syndrome. Diabetes 2001, 50:1844-1850.

2. Bloomgarden ZT: Second World Congress on the Insulin Resistance Syndrome: insulin resistance syndrome and nonalcoholic fatty liver disease. Diabetes Care 2005, 28:1518-1523.

3. Reaven GM: Banting lecture 1988. Role of insulin resistance in human disease. Diabetes 1988, 37:1595-1607.

4. Sheth SG, Gordon FD, Chopra S: Nonalcoholic steatohepatitis. Ann Intern Med 1997, 126:137-145.

5. Lupi R, Dotta F, Marselli L, Del GS, Masini M, Santangelo C, Patane G, Boggi U, Piro S, Anello M, et al: Prolonged exposure to free fatty acids has cytostatic and pro-apoptotic effects on human pancreatic islets: evidence that beta-cell death is caspase mediated, partially dependent on ceramide pathway, and Bcl-2 regulated. Diabetes 2002, 51:1437-1442.

6. Bak M, Kicinska-Krogulska M, Czerniak P, Michowicz A, Krakowiak A: [Toxic liver injuries-a current view on pathogenesis. Part II]. Med Pr 2011, 62:203-210.

7. Wang S, Kamat A, Pergola P, Swamy A, Tio F, Cusi K: Metabolic factors in the development of hepatic steatosis and altered mitochondrial gene expression in vivo. Metabolism 2011.

8. Mingrone G: Carnitine in type 2 diabetes. Ann N Y Acad Sci 2004, 1033:99-107.

9. Cave MC, Hurt RT, Frazier TH, Matheson PJ, Garrison RN, McClain CJ, McClave SA: Obesity, inflammation, and the potential application of pharmaconutrition. Nutr Clin Pract 2008, 23:16-34.

10. Broquist HP, Borum PR: Carnitine biosynthesis: nutritional implications. Adv Nutr Res 1982, 4:181-204.

11. Kerner J, Bieber L: Isolation of a malonyl-CoA-sensitive CPT/betaoxidation enzyme complex from heart mitochondria. Biochemistry 1990, 29:4326-4334.

12. Pearson DJ, Tubbs PK: Carnitine and derivatives in rat tissues. Biochem J 1967, 105:953-963.

13. Calabrese V, Cornelius C, Dinkova-Kostova AT, Calabrese EJ, Mattson MP: Cellular stress responses, the hormesis paradigm, and vitagenes: novel targets for therapeutic intervention in neurodegenerative disorders. Antioxid Redox Signal 2010, 13:1763-1811.

14. Rebouche $\mathrm{CJ}$, Seim $\mathrm{H}$ : Carnitine metabolism and its regulation in microorganisms and mammals. Annu Rev Nutr 1998, 18:39-61.

15. Broderick TL, Quinney HA, Lopaschuk GD: Carnitine stimulation of glucose oxidation in the fatty acid perfused isolated working rat heart. J Biol Chem 1992, 267:3758-3763.

16. Gramignano G, Lusso MR, Madeddu C, Massa E, Serpe R, Deiana L, Lamonica G, Dessi M, Spiga C, Astara G, et al: Efficacy of I-carnitine administration on fatigue, nutritional status, oxidative stress, and related quality of life in 12 advanced cancer patients undergoing anticancer therapy. Nutrition 2006, 22:136-145.

17. Mingrone G, Greco AV, Capristo E, Benedetti G, Giancaterini A, De GA Gasbarrini G: L-carnitine improves glucose disposal in type 2 diabetic patients. J Am Coll Nutr 1999, 18:77-82.

18. Beck-Nielsen $\mathrm{H}$ : Insulin resistance in skeletal muscles of patients with diabetes mellitus. Diabetes Metab Rev 1989, 5:487-493.

19. Lysiak W, Lilly K, DiLisa F, Toth PP, Bieber LL: Quantitation of the effect of $\mathrm{L}$-carnitine on the levels of acid-soluble short-chain acyl-CoA and CoASH in rat heart and liver mitochondria. J Biol Chem 1988, 263:1151-1156.

20. Rossignol DA, Frye RE: Mitochondrial dysfunction in autism spectrum disorders: a systematic review and meta-analysis. Mol Psychiatry 2011

21. Hao J, Shen W, Sun L, Long J, Sharman E, Shi X, Liu J: Mitochondrial dysfunction in the liver of type 2 diabetic Goto-Kakizaki rats: improvement by a combination of nutrients. Br J Nutr 2011, 1-8.

22. Karanth J, Jeevaratnam K: Effect of carnitine supplementation on mitochondrial enzymes in liver and skeletal muscle of rat after dietary lipid manipulation and physical activity. Indian J Exp Biol 2010, 48:503-510.

23. Bowyer BA, Miles JM, Haymond MW, Fleming CR: L-carnitine therapy in home parenteral nutrition patients with abnormal liver tests and low plasma carnitine concentrations. Gastroenterology 1988, 94:434-438. 
24. Romano M, Vacante M, Cristaldi E, Colonna V, Gargante MP, Cammalleri L, Malaguarnera M: L-carnitine treatment reduces steatosis in patients with chronic hepatitis C treated with alpha-interferon and ribavirin. Dig Dis Sci 2008, 53:1114-1121.

25. Malaguarnera M, Gargante MP, Russo C, Antic T, Vacante M,

Malaguarnera M, Avitabile T, Li VG, Galvano F: L-carnitine supplementation to diet: a new tool in treatment of nonalcoholic steatohepatitis-a randomized and controlled clinical trial. Am J Gastroenterol 2010, 105:1338-1345.

26. Tisi A, Federico R, Moreno S, Lucretti S, Moschou PN, RoubelakisAngelakis KA, Angelini R, Cona A: Perturbation of polyamine catabolism can strongly affect root development and xylem differentiation. Plant Physiol 2011, 157:200-215.

27. Cao Y, Wang YX, Liu CJ, Wang LX, Han ZW, Wang CB: Comparison of pharmacokinetics of $\mathrm{L}$-carnitine, acetyl-L-carnitine and propionyl-Lcarnitine after single oral administration of L-carnitine in healthy volunteers. Clin Invest Med 2009, 32:E13-E19.

28. Brunt EM, Tiniakos DG: Histopathology of nonalcoholic fatty liver disease. World J Gastroenterol 2010, 16:5286-5296.

29. Luo J, Quan J, Tsai J, Hobensack CK, Sullivan C, Hector R, Reaven GM: Nongenetic mouse models of non-insulin-dependent diabetes mellitus. Metabolism 1998, 47:663-668.

30. James O, Day C: Non-alcoholic steatohepatitis: another disease of affluence. Lancet 1999, 353:1634-1636.

31. Kiens B, Essen-Gustavsson B, Gad P, Lithell H: Lipoprotein lipase activity and intramuscular triglyceride stores after long-term high-fat and highcarbohydrate diets in physically trained men. Clin Physiol 1987, 7:1-9.

32. RANDLE PJ, GARLAND PB, HALES CN, NEWSHOLME EA: The glucose fattyacid cycle. Its role in insulin sensitivity and the metabolic disturbances of diabetes mellitus. Lancet 1963, 1:785-789.

33. Bloomgarden ZT: Second World Congress on the Insulin Resistance Syndrome: insulin resistance syndrome and nonalcoholic fatty liver disease. Diabetes Care 2005, 28:1518-1523.

34. Purohit V, Rapaka R, Shurtleff D: Role of cannabinoids in the development of fatty liver (steatosis). AAPS J 2010, 12:233-237.

35. Lupi R, Dotta F, Marselli L, Del GS, Masini M, Santangelo C, Patane G, Boggi U, Piro S, Anello M, et al: Prolonged exposure to free fatty acids has cytostatic and pro-apoptotic effects on human pancreatic islets: evidence that beta-cell death is caspase mediated, partially dependent on ceramide pathway, and Bcl-2 regulated. Diabetes 2002, 51:1437-1442.

36. Ahmad S: L-carnitine in dialysis patients. Semin Dial 2001, 14:209-217.

37. Srinivas SR, Prasad PD, Umapathy NS, Ganapathy V, Shekhawat PS: Transport of butyryl-L-carnitine, a potential prodrug, via the carnitine transporter OCTN2 and the amino acid transporter $\operatorname{ATB}(0,+)$. Am J Physiol Gastrointest Liver Physiol 2007, 293:G1046-G1053.

38. Cha YS: Effects of L-carnitine on obesity, diabetes, and as an ergogenic aid. Asia Pac J Clin Nutr 2008, 17(Suppl 1):306-308.

39. McGarry JD, Robles-Valdes C, Foster DW: Role of carnitine in hepatic ketogenesis. Proc Natl Acad Sci USA 1975, 72:4385-4388.

40. Coleman RA, Lee DP: Enzymes of triacylglycerol synthesis and their regulation. Prog Lipid Res 2004, 43:134-176.

41. Pons R, De Vivo DC: Primary and secondary carnitine deficiency syndromes. J Child Neurol 1995, 10(Suppl 2):S8-24.

42. Scholte HR, Rodrigues PR, de Jonge PC, Luyt-Houwen IE, Hedwig M, Verduin M, Ross JD: Primary carnitine deficiency. J Clin Chem Clin Biochem 1990, 28:351-357.

43. Ames BN: Delaying the mitochondrial decay of aging. Ann N Y Acad Sci 2004, 1019:406-411.

doi:10.1186/1758-5996-3-31

Cite this article as: Xia et al:: L-carnitine ameliorated fatty liver in highcalorie diet/STZ-induced type 2 diabetic mice by improving mitochondrial function. Diabetology \& Metabolic Syndrome 2011 3:31.

\section{Submit your next manuscript to BioMed Central and take full advantage of:}

- Convenient online submission

- Thorough peer review

- No space constraints or color figure charges

- Immediate publication on acceptance

- Inclusion in PubMed, CAS, Scopus and Google Scholar

- Research which is freely available for redistribution

Submit your manuscript at www.biomedcentral.com/submit
Biomed Central 